\title{
FLAME RETARDANTS EFFECTS ON THE INITIAL GROWTH OF Schizolobium amazonicum HUBER EX DUCKE
}

\author{
Elen Silma Oliveira Cruz Ximenes ${ }^{2 *}$, Andréa Carvalho da Silva $^{2}$, $^{\text {, Adilson Pacheco de Souza }}$ A $^{\circ}$, \\ Josiane Fernandes Keffer ${ }^{4}\left(\right.$,Alison Martins dos Anjos ${ }^{5} \odot$ and Felipe Gomes Costa ${ }^{5}($
}

\footnotetext{
${ }^{1}$ Received on 02.03.2020 accepted for publication on 19.02.2021.

${ }^{2}$ Universidade Federal de Mato Grosso, Programa de Pós-Graduação em Agronomia, Sinop, MT - Brasil. E-mail: <elenxflo@gmail. com>and <andcar1007@gmail.com>.

${ }^{3}$ Universidade Federal de Mato Grosso, Instituto de Ciências Agrárias e Ambientais, Sinop, MT - Brasil. E-mail: < pachecoufmt@gmail. com>.

${ }^{4}$ Universidade Federal de Mato Grosso, Programa de Pós-Graduação em Ciências Ambientais, Sinop, MT - Brasil. E-mail: <kefferjf@ gmail.com>.

${ }^{5}$ Universidade Federal de Mato Grosso, Graduando em Agronomia, Sinop, MT - Brasil. E-mail: <alison.martins102@gmail.com>and $<$ felipecosta.gc@gmail.com>

*Corresponding author.
}

\begin{abstract}
Flame retardants are efficient in fighting wildfire; however, their environmental implications, especially regarding the vegetation, need to be clarified. This work aimed at assessing the effects of flame retardant on the initial growth of Schizolobium amazonicum. Treatments consisted in applying different flame retardant concentrations via substrate and leaf: Phos-Chek WD- $881^{\circledR}\left(0,3.00,6.00,8.00\right.$ and $\left.10.00 \mathrm{~mL} \mathrm{~L}^{-1}\right)$, Hold Fire $^{\circledR}\left(0,7.00,9.00,12.00\right.$ and $\left.15.00 \mathrm{~mL} \mathrm{~L}^{-1}\right)$ and water-retaining polymer Nutrige ${ }^{\circledR}$ used as alternative retardant $\left(0,0.25,0.50,0.75\right.$ and $\left.1.00 \mathrm{~g} \mathrm{~L}^{-1}\right)$. Growth analyses were carried out to assess the effects of these substances (10 repetitions per treatment). The aliquot of $10.00 \mathrm{~mL} \mathrm{~L}^{-1}$ of Phos-Chek WD881 applied on the leaves led to an increase of $70 \%$ in leaf area and $15 \%$ in seedling height. The same Phos-Chek concentration favored height increase (32\%) and total dry mass accumulation (33\%) throughout time. The concentration of $15 \mathrm{~mL} \mathrm{~L}^{-1}$ of Hold Fire ${ }^{\circledR}$ applied on leaves, compromised $45 \%$ the accumulation of dry biomass in the seedling. Initially, $1.00 \mathrm{~g} \mathrm{~L}^{-1}$ of Nutrige ${ }^{\circledR}$ applied via substrate led to an increase of $70 \%$ in leaf area, $29 \%$ in plant height, and $89 \%$ in leaf dry mass. Therefore, Phos-Chek applied on leaves favored shoot growth in S. amazonicum. Hold Fire ${ }^{\circledR}$ applied on leaves impaired biomass accumulation in seedlings. Nutrigel ${ }^{\circledR}$ applied on substrate does not cause long-lasting damage to the initial growth of $S$. amazonicum. The aliquot of $0.50 \mathrm{~g} \mathrm{~L}^{-1}$ administered via polymer leave had positive effect on seedling shoot.
\end{abstract}

Keywords: Wildfire; Hydrogel; Growth analysis.

\section{EFEITOS DE RETARDANTES DE FOGO SOBRE O CRESCIMENTO INICIAL DE Schizolobium amazonicum HUBER EX DUCKE}

RESUMO - Retardantes de fogo são eficazes em combater incêndios florestais, contudo, suas implicações ambientais, principalmente quanto à vegetação, precisam ser melhor esclarecidas. Neste trabalho, objetivouse avaliar os efeitos de retardantes de fogo sobre o crescimento inicial de Schizolobium amazonicum. Os tratamentos consistiram em aplicações via substrato e foliar de concentrações dos retardantes de fogo: PhosChek WD-881 ${ }^{\circledR}\left(0 ; 3,00 ; 6,00 ; 8,00\right.$ e 10,00 mL L $\left.{ }^{-1}\right)$; Hold Fire ${ }^{\circledR}\left(0 ; 7,00 ; 9,00 ; 12,00 ;\right.$ e 15,00 $\left.\mathrm{mL} \mathrm{L} \mathrm{L}^{-1}\right)$ e do polímero hidroretentor Nutrige ${ }^{\circledR}$ usado como retardante alternativo $\left(0 ; 0,25 ; 0,50 ; 0,75\right.$ e $\left.1,00 \mathrm{~g} \mathrm{~L}^{-1}\right)$. Para avaliação dos efeitos realizaram-se análises de crescimento, com 10 repetições por tratamento. Compararamse as médias pelo Teste de Tukey $(p<0,05)$ conforme significância da análise de variância. A concentração de $10,00 \mathrm{~mL} \mathrm{~L} \mathrm{~L}^{-1}$ de Phos-Chek WD881 via foliar proporcionou incrementos na área foliar (71\%) e altura (18\%) das mudas. No decorrer do tempo, a mesma concentração de Phos-Chek favoreceu o crescimento em altura (32\%) e acúmulo de massa seca total (33\%). A concentração de $15 \mathrm{~mL} \mathrm{~L}^{-1}$ de Hold Fire ${ }^{\circledR}$ via foliar comprometeu em 45\% o acúmulo de biomassa seca das mudas. Inicialmente, $1,00 \mathrm{~g} \mathrm{~L}^{-1}$ de Nutrigel ${ }^{\circledR}$ via substrato diminuiu em $28 \%$ a média de massa seca. A concentração $0,50 \mathrm{~g} \mathrm{~L}^{-1}$ do polímero via foliar do polímero promoveu incrementos na área foliar (70\%), altura (29\%) e massa seca das folhas (89\%). Portanto, Phos-Chek via foliar

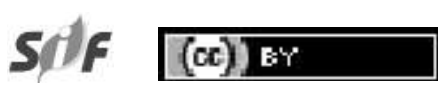

Revista Árvore 2021;45:e4514 http://dx.doi.org/10.1590/1806-908820210000014 
favoreceu o crescimento da parte aérea de $\boldsymbol{S}$. amazonicum. Hold Fire ${ }^{\circledR}$ via foliar prejudicou o acúmulo de biomassa das mudas. Nutrigel ${ }^{\circledR}$ via substrato não provocou prejuizos duradouros sobre o crescimento inicial de $\boldsymbol{S}$. amazonicum. 0,50 $\mathrm{g} \mathrm{L}^{-1}$ via foliar do polímero teve efeito positivo sobre o crescimento da parte aérea das mudas.

Palavras-Chave: Incêndio florestal; Hidrogel; Análises de Crescimento.

\section{INTRODUCTION}

Concern with ecological, economic and social damage caused by wildfire has boosted the development of promising techniques to prevent and hold fire, and flame retardant using stands out among them. Retardants are chemical products added to water to increase its extinguishing efficiency by reducing time and the amount of water resources necessary to control fire (Couto-Vazquéz and Gonzaléz-Prieto, 2013).

Flame retardants can have long or short duration depending on the time their extinguishing behavior lasts. Overall, long duration retardants have fertilizing salts that keep the suppressing activity even after water evaporation (Kalabokidis, 2000; Giménez et al., 2004; Marshal et al., 2016). Short duration retardants, or foam fire suppression, are composed of surfactant, foaming and wetting agents that change water physical features by delaying evaporation and producing a continuous coat over the combustible material (Plucinski et al., 2017).

If one takes into account wildfire events in Brazil, and their influence on significant environmental changes (Aragão et al., 2018; Silva et al., 2018), flame retardants can become an ally to mitigate losses caused by uncontrolled fire. However, the use of these substances remains scarce in the Brazilian territory, since most products available in the market are imported, a fact that impairs their use due to their high prices, lack of information about them and of a legislation to regulate their use (Machado Filho et al., 2012). Few national research about this topic are related to retardants' efficiency in fighting fire (Machado Filho et al., 2012; Fiedler et al., 2015; Canzian, et al., 2016).

On the other hand, water-retaining polymers known in the agroforestry sector as soil conditioners have been tested as sustainable and economically feasible alternatives to hold flames (Souza et al., 2012; Lima et al., 2020a,b). Hydrogel using in agriculture is a sustainable, ecological and promising technology (Azevedo et al., 2016; Kozen et al., 2017; Pontes Filho et al., 2018). Water-retaining polymers can be an alternative to hold fire, since they keep the combustible material moistened by increasing water viscosity. The anti-fire application of such products can be safer for the environment because of the legislation that regulates their use, which takes into account their action as soil conditioners.

Research focusing on the investigation about the effects of flame-retardant effects on the environment are necessary to join fire-fighting efficiency to protect forest ecosystems, mainly when it comes to remnant vegetation behavior. It is so, because litter receives and stores the applied broth in case of fire extinguishing.

The forest species Schizolobium amazonicum Huber ex Ducke, popularly known as 'paricá' or 'pinho-cuiabano', is economically and ecologically relevant for the Amazonian region. This species has the potential to compose agroforestry systems and reforestation programs given its fast growth (Cordeiro et al., 2015). Thus, the present study aimed at assessing the effects of different flame-retardant concentrations and of a water-retaining polymer on the initial growth phase of individuals belonging to species $S$. amazonicum.

\section{MATERIALS AND METHODS}

The experiment was conducted in greenhouse between July and October 2018, during the dry season in the Cerrado-Amazon transition region (Souza et al., 2013). The greenhouse had curved roof, transparent PEBD (120-microns) plastic coating and its sides were covered with $50 \%$ black polyester fabric. Its dimensions were $12 \mathrm{~m}$ in length, $6 \mathrm{~m}$ in width and $4.5 \mathrm{~m}$ in height; it headed Northeast.

S. amazonicum seeds were collected from urban afforestation matrices (11 50 ' $48.39^{\prime \prime} \mathrm{S}, 55^{\circ} 31^{\prime} 30.42^{\prime \prime}$ W; $\left.11^{\circ} 51^{\prime} 4.95^{\prime \prime} \mathrm{S}, 55^{\circ} 31^{\prime} 17.11^{\prime \prime} \mathrm{W}\right)$. Half of the seeds germinated in polypropylene tubes, in greenhouse

Revista Árvore 2021;45:e4514 
environment, and were treated with substrate since their sowing in order to avoid the effects of retardant application procedures. The other half of them germinated inside germination chamber, on plastic trays coated with germitest paper, under constant temperature $\left(25^{\circ} \mathrm{C}\right)$ and $12-\mathrm{h}$ photoperiod. Seedlings from the germination chamber were transferred to tubes filled with substrate after root protrusion and cotyledons' expansion. They remained in greenhouse and were treated via leaf, after transplantation.

Two tube volumes were used in the experiment, depending on seedlings' evaluation time: $820 \mathrm{~cm}^{3}$, for seedlings assessed at the end of the experiment ( 80 DAS/DAT) and $290 \mathrm{~cm}^{3}$ for the other ones. Substrate composition consisted of the following mix: 2 forest soil (Dystrophic red-yellow latosol): 1 commercial organic compound (bio-stabilized pine bark, vermiculite, charcoal grinder and phenolic foam).

Treatments comprised different concentrations of short duration flame retardant (Phos-Chek WD$881^{\circledR}$ and Hold Fire ${ }^{\circledR}$ ) and Nutrigel ${ }^{\circledR}$ water-retaining polymer. These products were selected based on their commercial use and availability in Brazil. Products were not compared to each other, since they presented different chemical compositions and behaviors. Product concentrations were based on prescribed burns conducted by Lima et al. (2020b) in a site of Eucalyptus urograndis Clone H13. It was done to assess fire behavior and product/concentration/time efficiency after spray application.

Retardant Phos-Chek WD881 ${ }^{\circledR}$ (ICL Performance Products LP, St. Louis, MO, EUA) is composed of alpha olefin sulfonate, 2-methylpentane-2,4-diol, lauryl alcohol d-limonene. The following concentrations were set based on the dilution limits recommended by the manufacturer (from $0.1 \%$ to $1 \%$ ): $0.00,3.00,6.00$, 8.00 and $10.00 \mathrm{~mL} \mathrm{~L}^{-1}$. The Brazilian product Hold Fire $^{\circledR}$ (Favaro \& Perin Ind. and Com. LTDA - ME, Vila Velha, ES, BR) is offered in the form of viscous gel; it is composed of vegetal oils, hygroscopic natural polymer and biodegradable surfactants. Mix dose recommended by the manufacturer ranges from $0.7 \%$ to $1.5 \%$; therefore, the following concentrations were used: $0.00,7.00,9.00,12.00$ and $15.00 \mathrm{~mL} \mathrm{~L}^{-1}$.

Nutrigel $^{\circledR}$ (Agroterra Insumos, São José do Rio Preto, SP, BR) consists of water-retaining polymer specially developed for agricultural use as soil conditioner and water absorber. The maximum amount of water-retaining polymer - applicable with fan-type nozzle backpack pump used for fire-fighting purposes - was determined through field assays conducted to test it as alternative flame retardant. Thus, the maximum concentration of $1.00 \mathrm{~g} \mathrm{~L}^{-1}$ was adopted to avoid clogging - it allowed the following fractionation: $0.00,0.25,0.50,0.75$ and $1.00 \mathrm{~g} \mathrm{~L}^{-1}$.

Both the retardants and water-retaining polymer were applied fortnight from the experiment installation day on, always in the morning, in order to replace irrigation. Syringes $(20 \mathrm{~mL})$ were used to administer the treatment via substrate, which was manually sprayed on the leaves. Only water was used in treatments based on concentrations equal to zero (0) - control treatment.

Destructive growth analyses were applied to $S$. amazonicum seeds at 15,30,60 and 80 days after sowing (DAS) and transplanting (DAT), with 10 repetitions per treatment, in order to assess retardants and water-retaining polymer's effects. The following growth variables were assessed: Number of leaves (LN) and leaflets (NL) (visual counting), leaf area per plant (LA) (photoelectric meter L3000, Li-cor Company, Nebraska, USA), collar diameter (D) (at substrate height - with the aid of digital caliper), seedling height $(\mathrm{H})$ (from collar to apical bud - with the aid of scaled ruler), root volume (RV) (graduated cylinder through washed roots' immersion in known water volume), dry mass of each plant partition (leaves (LDM), stem (SDM) and root (RDM)) and total (TDM) (in analytical scale, at precision of $0.0001 \mathrm{~g}$, after vegetal material drying in forced air circulation oven at $65^{\circ} \mathrm{C} \pm 2{ }^{\circ} \mathrm{C}$ for 72 hours).

The experiment followed a completely randomized design. Statistical analyses were carried out in separate, for each application form (via substrate or leaf application) at the beginning (15 DAS or DAT) and at the end of the experiment (80 DAS or DAT). Data were assessed when they were proved normal through the Shapiro-Wilk test, at $5 \%$ significance level; they were changed through the function $\mathrm{Y}=(\mathrm{x}+1)^{0.5}$ when they were significant (non-normal). Means recorded for the variables were presented in their original form; statistics referred to transformed data. Analysis of variance was carried out and significant means $(p<0.05)$ were compared through Tukey test at 5\% probability level.

Revista Árvore 2021;45:e4514 


\section{RESULTS}

\subsection{Phos-Chek WD881 ${ }^{\circledR}$ via substrate}

None of the assessed variables for $S$. amazoncium under Phos-Chek WD88 $1{ }^{\circledR}$ concentrations administered via substrate have recorded any difference.

\subsection{Phos-Chek WD881 ${ }^{\circledR}$ via leaf}

There were differences between witness concentration $\left(0.00 \mathrm{ml} \mathrm{L}^{-1}\right)$ and maximum PhosChek WD881 ${ }^{\circledR}\left(10.00 \mathrm{ml} \mathrm{L}^{-1}\right)$ application via leaf in variables LA and $\mathrm{H}$ at 15 DAT. Mean LA at concentrations $0.00 ; 6.00$ and $8.00 \mathrm{ml} \mathrm{L}^{-1}$ were statistically similar to each other and lower than those at retardant concentration of $10.00 \mathrm{ml} \mathrm{L}^{-1}$. The mean recorded for the LA treatment $3.00 \mathrm{ml} \mathrm{L}^{-1}$ of PhosChek WD881 ${ }^{\circledR}$ did not present differences in relation to the other treatments $(0.00 ; 6.00 ; 8.00$ and 10.00 $\left.\mathrm{ml} \mathrm{L}^{-1}\right)$. Mean concentration of $10.00 \mathrm{ml} \mathrm{L}^{-1}$ of PhosChek WD88 $1^{\circledR}$ via leaf was higher in variable $H$ than that of treatments $0.00 ; 3.00$ and $6.00 \mathrm{ml} \mathrm{L}^{-1}$. Mean $\mathrm{H}$ in treatment $8.00 \mathrm{ml} \mathrm{L}^{-1}$ did not differ from the other ones $\left(0.00 ; 3.00 ; 6.00\right.$ and $\left.10.00 \mathrm{ml} \mathrm{L}^{-1}\right)$ (Table 1$)$.

The highest Phos-Chek WD881 ${ }^{\circledR}(10.00 \mathrm{~mL}$ $\left.\mathrm{L}^{-1}\right)$ concentration applied on leaves led to $41.45 \mathrm{~cm}^{2}$
(71\%) LA and $5.22 \mathrm{~cm} \mathrm{(18 \% )} \mathrm{H} \mathrm{increase} \mathrm{at} 15$ DAT when the means of these variables were compared to the concentration applied to the control (Table 1).

There were no significant differences between means recorded for treatments 8.00 and $10.00 \mathrm{ml} \mathrm{L}^{-1}$ of Phos-Chek WD88 $1^{\circledR}$ via leaf in variables $\mathrm{D}, \mathrm{H}, \mathrm{RV}$, LDM, SDM, RDM and TDM at 80 DAT. However, the mean recorded for the treatment without retardant (0.00) was lower than at least one among treatments with retardant in variable $\mathrm{NL}, \mathrm{H}$ and TDM. Means recorded for variables LA, D, RV LDM, SDM and RDM in the witness treatment (0.00) did not differ from those recorded for the highest concentrations of Phos-Chek WD881 ${ }^{\circledR}\left(10.00 \mathrm{ml} \mathrm{L}^{-1}\right)$ (Table 1).

Phos-Chek WD881 ${ }^{\circledR}$ concentration of $10 \mathrm{ml} \mathrm{L}^{-1}$ applied on leaves favored $\mathrm{H}$ increase by $4.8 \mathrm{~cm} \mathrm{(32}$ $\%)$ and TDM accumulation increase by $0.48 \mathrm{~g} \mathrm{(33 \% )}$ in comparison to the means recorded for the control concentration at 80 DAT (Table 1).

\subsection{Hold Fire ${ }^{\circledR}$ via substrate}

Hold Fire ${ }^{\circledR}$ concentrations administered via substrate were only effective for $S$. amazonicum growth at 15 DAS. Seedlings' mean $\mathrm{H}$ was $1.96 \mathrm{~cm}(16 \%)$ higher at Hold Fire ${ }^{\circledR}$ concentration of $12.00 \mathrm{ml} \mathrm{L}^{-1}$

Table 1 - Mean growth values recorded for variables of seedlings belonging to species S. amazonicum subjected to different Phos-Chek WD881 ${ }^{\circledR}$ concentrations - applied on leaves.

Tabela 1 - Valores médios das variáveis de crescimento das mudas da espécie $\boldsymbol{S}$. amazonicum, submetidas a diferentes concentrações do retardante Phos-Chek WD881 ${ }^{\circledR}$ - aplicadas sobre as folhas.

\begin{tabular}{|c|c|c|c|c|c|c|c|c|c|c|}
\hline Conc. & $\mathbf{L N}$ & $\mathbf{N L}$ & $\mathbf{L A}$ & $\overline{\mathbf{D}}$ & $\overline{\mathbf{H}}$ & $\overline{\mathbf{R V}}$ & LDM & SDM & RDM & $\overline{\text { TDM }}$ \\
\hline & - & - & $\left(\mathrm{cm}^{2}\right)$ & $(\mathrm{mm})$ & $(\mathrm{cm})$ & $(\mathrm{ml})$ & \multicolumn{4}{|c|}{ 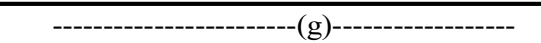 } \\
\hline \multicolumn{11}{|c|}{15 DAT } \\
\hline 0.00 & $4.90 \mathrm{a}$ & $74.70 \mathrm{a}$ & $58.40 \mathrm{~b}$ & $2.78 \mathrm{a}$ & $13.77 \mathrm{~b}$ & $1.05 \mathrm{a}$ & $0.19 \mathrm{a}$ & $0.16 \mathrm{a}$ & $0.09 \mathrm{a}$ & $0.43 \mathrm{a}$ \\
\hline 3.00 & $4.30 \mathrm{a}$ & $73.10 \mathrm{a}$ & $66.46 \mathrm{ab}$ & $2.80 \mathrm{a}$ & $14.59 \mathrm{~b}$ & $1.20 \mathrm{a}$ & $0.20 \mathrm{a}$ & $0.15 \mathrm{a}$ & $0.10 \mathrm{a}$ & $0.45 \mathrm{a}$ \\
\hline 6.00 & $3.60 \mathrm{a}$ & $60.70 \mathrm{a}$ & $58.55 \mathrm{~b}$ & $2.71 \mathrm{a}$ & $15.85 \mathrm{~b}$ & $1.25 \mathrm{a}$ & $0.17 \mathrm{a}$ & $0.18 \mathrm{a}$ & $0.09 \mathrm{a}$ & $0.44 \mathrm{a}$ \\
\hline 8.00 & $4.50 \mathrm{a}$ & $65.30 \mathrm{a}$ & $59.26 \mathrm{~b}$ & $2.83 \mathrm{a}$ & $14.01 \mathrm{ab}$ & $1.10 \mathrm{a}$ & $0.18 \mathrm{a}$ & $0.15 \mathrm{a}$ & $0.09 \mathrm{a}$ & $0.42 \mathrm{a}$ \\
\hline 10.00 & $5.40 \mathrm{a}$ & $86.50 \mathrm{a}$ & $99.85 \mathrm{a}$ & $3.06 \mathrm{a}$ & $18.99 \mathrm{a}$ & $1.60 \mathrm{a}$ & $0.28 \mathrm{a}$ & $0.23 \mathrm{a}$ & $0.15 \mathrm{a}$ & $0.66 \mathrm{a}$ \\
\hline$\overline{\mathrm{MSD}}$ & 2.82 & 40.86 & 38.26 & 0.39 & 3.55 & 0.65 & 0.13 & 0.09 & 0.06 & 0.25 \\
\hline \multicolumn{11}{|c|}{80 DAT } \\
\hline 0.00 & $9.50 \mathrm{a}$ & $127.70 \mathrm{~b}$ & $57.31 \mathrm{ab}$ & $4.06 \mathrm{ab}$ & $14.98 \mathrm{bc}$ & $2.50 \mathrm{ab}$ & $0.35 \mathrm{ab}$ & $0.54 \mathrm{ab}$ & $0.54 \mathrm{abc}$ & $1.43 \mathrm{~b}$ \\
\hline 3.00 & $11.17 \mathrm{a}$ & $150.67 \mathrm{ab}$ & $48.99 \mathrm{ab}$ & $3.372 \mathrm{~b}$ & $12.60 \mathrm{~b}$ & $1.08 \mathrm{c}$ & $0.28 \mathrm{ab}$ & $0.32 \mathrm{c}$ & $0.30 \mathrm{c}$ & $0.91 \mathrm{ab}$ \\
\hline 6.00 & $11.63 \mathrm{a}$ & $161.37 \mathrm{ab}$ & $39.19 \mathrm{~b}$ & $3.62 \mathrm{ab}$ & $15.89 \mathrm{c}$ & $1.69 \mathrm{bc}$ & $0.25 \mathrm{~b}$ & $0.54 \mathrm{bc}$ & $0.43 \mathrm{bc}$ & $1.23 \mathrm{ab}$ \\
\hline 8.00 & $12.30 \mathrm{a}$ & $182.85 \mathrm{a}$ & $69.84 \mathrm{a}$ & $4.01 \mathrm{ab}$ & $17.15 \mathrm{ab}$ & $3.18 \mathrm{a}$ & $0.38 \mathrm{ab}$ & $0.73 \mathrm{a}$ & $0.60 \mathrm{ab}$ & $1.71 \mathrm{a}$ \\
\hline 10.00 & $10.55 \mathrm{a}$ & $150.85 \mathrm{ab}$ & $58.16 \mathrm{ab}$ & $4.72 \mathrm{a}$ & $19.78 \mathrm{a}$ & $2.61 \mathrm{ab}$ & $0.39 \mathrm{a}$ & $0.77 \mathrm{a}$ & $0.74 \mathrm{a}$ & $1.91 \mathrm{a}$ \\
\hline$\overline{M S D}$ & 3.47 & 49.6 & 26.41 & 0.89 & 3.47 & 1.3 & 0.14 & 0.36 & 0.27 & 0.69 \\
\hline \multicolumn{11}{|c|}{$\begin{array}{l}\text { Means followed by the same letter did not differ from each other in the Tukey's test at 5\% probability level. Conc: concentrations; LN: number of leaves; NL: number } \\
\text { of leaflets; LA: leaf area; D: collar diameter; H: height; RV: root volume; SDM: stem dry mass; RDM: root dry mass; LDM: leaf dry mass; TDM: total dry mass; } \\
\text { MSD: minimal significant difference. } \\
\text { Médias seguidas pela mesma letra não diferem entre si pelo teste de Tukey a } 5 \% \text { de probabilidade. NF: número de folhas; NFO: número de foliolos; AF: área foliar; } \\
\text { D: diâmetro do coleto; H: altura; VR: volume de raiz; MSC: massa seca do caule; MSR: massa seca de raiz; MSF: massa seca das folhas; MST: massa seca total; } \\
\text { DMS: diferença minima significativa. }\end{array}$} \\
\hline
\end{tabular}

Revista Árvore 2021;45:e4514 
applied via substrate than that recorded for the control. However, outcomes of such a treatment did not differ from those at concentrations $7.00 ; 9.00$ and $15.00 \mathrm{ml} \mathrm{L}^{-1}$. The mean RV recorded for seedlings only treated with water $\left(0.00 \mathrm{ml} \mathrm{L}^{-1}\right.$ of Hold Fire $\left.{ }^{\circledR}\right)$ did not statistically differ from that of concentrations with retardant. The lowest tested Hold Fire ${ }^{\circledR}$ concentrations (7.00 and 9.00 $\mathrm{ml} \mathrm{L}^{-1}$ ) led to mean $\mathrm{RV}$ higher than that of the treatment based on $15 \mathrm{ml} \mathrm{L}^{-1}$ of retardant (Table 2).

\subsection{Hold Fire ${ }^{\circledR}$ via leaf}

The treatment based on $12.00 \mathrm{ml} \mathrm{L}^{-1}$ of Hold Fire $^{\circledR}$ via leaf did not present differences concerning the highest retardant concentration $\left(15.00 \mathrm{ml} \mathrm{L}^{-1}\right)$ in variable D at $15 \mathrm{DAT}$, but it was higher than that of treatments based on $0.00,7.00$ and $9.00 \mathrm{ml} \mathrm{L}^{-1}$ of Hold Fire $^{\circledR}$. Mean D was $0.55 \mathrm{~mm}(20 \%)$ wider at 15 DAT than that of the control due to the application of 12.00 $\mathrm{ml} \mathrm{L}{ }^{-1}$ of Hold Fire ${ }^{\circledR}$ via substrate (Table 3 ).

Mean $\mathrm{H}$ recorded for the treatment without retardant $\left(0.00 \mathrm{ml} \mathrm{L}^{-1}\right)$ did not differ from that with concentrations with it $(7.00 ; 9.00 ; 12.00$ and $15.00 \mathrm{ml}$ $\left.\mathrm{L}^{-1}\right)$ at 15 DAT. The mean recorded for this variable in treatment $7.00 \mathrm{ml} \mathrm{L}^{-1}$ was higher than that of treatments based on 12.00 and $15.00 \mathrm{ml} \mathrm{L}^{-1}$ of Hold Fire $^{\circledR}$ via leaf (Table 3 ).
Seedlings treated with water $\left(0.00 \mathrm{ml} \mathrm{L}^{-1}\right.$ of Hold Fire $^{\circledR}$ ) presented D, SDM and TDM means higher than those of the treatment based on $15.00 \mathrm{ml} \mathrm{L}^{-1}$ of retardant, at 80 DAT. However, these variables did not present differences between the control treatment and that with retardant concentration of $9 \mathrm{ml} \mathrm{L}^{-1}$. The mean recorded for variable $\mathrm{H}$ without Hold Fire ${ }^{\circledR}(0.00 \mathrm{ml}$ $\left.\mathrm{L}^{-1}\right)$ application did not change in comparison to the other treatments. Mean RV of seedlings that were not treated with retardant $\left(0.00 \mathrm{ml} \mathrm{L}^{-1}\right)$ was similar to that of treatments based on concentration of $15.00 \mathrm{ml}$ $\mathrm{L}^{-1}$. These two treatments were better than treatments based on 7.00 and $12.00 \mathrm{ml} \mathrm{L}^{-1}$, although they did not differ from the one presenting $9.00 \mathrm{ml} \mathrm{L}^{-1}$ Hold Fire ${ }^{\circledR}$ (Table 3).

The highest Hold Fire ${ }^{\circledR}$ concentration $(15 \mathrm{~mL}$ $\left.\mathrm{L}^{-1}\right)$ applied to leaves highlighted decrease by 0.85 $\mathrm{mm}(21 \%)$ in mean D; by $0.30 \mathrm{~g}(55 \%)$ in SDM and by $0.64 \mathrm{~g}(45 \%)$ in the TDM of $S$. amazonicum seedlings, at $80 \mathrm{DAT}$, when they were compared to the growth rates recorded for these variables at control concentrations (Table 3).

\subsection{Nutrige ${ }^{\circledR}$ via substrate}

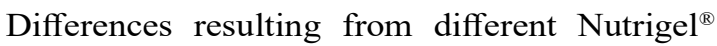
concentrations applied via substrate were only observed at 15 DAS.

Table 2 - Mean growth values recorded for variables of seedlings belonging to species S. amazonicum subjected to different Phos-Chek WD881 ${ }^{\circledR}$ concentrations - applied via substrate.

Tabela 2 - Valores médios das variáveis de crescimento das mudas da espécie $\boldsymbol{S}$. amazonicum, submetidas a diferentes concentrações do retardante Hold Fire ${ }^{\circledR}$ - aplicadas via substrato.

\begin{tabular}{|c|c|c|c|c|c|c|c|c|c|c|}
\hline Conc. & $\mathbf{L N}$ & $\mathbf{N L}$ & $\overline{\mathbf{L A}}$ & $\overline{\mathbf{D}}$ & $\overline{\mathbf{H}}$ & $\mathbf{R V}$ & LDM & SDM & RDM & $\overline{\text { TDM }}$ \\
\hline & - & - & $\mathrm{cm}^{2}$ & $\mathrm{~mm}$ & $\mathrm{~cm}$ & $\mathrm{ml}$ & \multicolumn{4}{|c|}{---------------------------g---------------------- } \\
\hline \multicolumn{11}{|c|}{15 DAS } \\
\hline 0.00 & $2.40 \mathrm{a}$ & $44.40 \mathrm{a}$ & $78.08 \mathrm{a}$ & $3.33 \mathrm{a}$ & $10.41 \mathrm{~b}$ & $1.90 \mathrm{ab}$ & $0.17 \mathrm{a}$ & $0.25 \mathrm{a}$ & $0.11 \mathrm{a}$ & $0.53 \mathrm{a}$ \\
\hline 7.00 & $2.70 \mathrm{a}$ & $50.20 \mathrm{a}$ & $84.78 \mathrm{a}$ & $3.53 \mathrm{a}$ & $12.14 \mathrm{ab}$ & $2.20 \mathrm{a}$ & $0.19 \mathrm{a}$ & $0.31 \mathrm{a}$ & $0.10 \mathrm{a}$ & $0.60 \mathrm{a}$ \\
\hline 9.00 & $2.70 \mathrm{a}$ & $50.00 \mathrm{a}$ & $95.12 \mathrm{a}$ & $3.59 \mathrm{a}$ & $10.94 \mathrm{ab}$ & $2.40 \mathrm{a}$ & $0.21 \mathrm{a}$ & $0.26 \mathrm{a}$ & $0.13 \mathrm{a}$ & $0.60 \mathrm{a}$ \\
\hline 12.00 & $2.80 \mathrm{a}$ & $53.80 \mathrm{a}$ & $97.85 \mathrm{a}$ & $3.65 \mathrm{a}$ & $12.37 \mathrm{a}$ & $1.90 \mathrm{ab}$ & $0.21 \mathrm{a}$ & $0.28 \mathrm{a}$ & $0.12 \mathrm{a}$ & $0.62 \mathrm{a}$ \\
\hline 15.00 & $2.70 \mathrm{a}$ & $52.40 \mathrm{a}$ & $74.65 \mathrm{a}$ & $3.71 \mathrm{a}$ & $11.63 \mathrm{ab}$ & $1.30 \mathrm{~b}$ & $0.18 \mathrm{a}$ & $0.26 \mathrm{a}$ & $0.11 \mathrm{a}$ & $0.56 \mathrm{a}$ \\
\hline MSD & 0.61 & 11.15 & $27.90 \mathrm{a}$ & 0.48 & 1.81 & 0.75 & 0.07 & 0.14 & 0.03 & 0.16 \\
\hline \multicolumn{11}{|c|}{80 DAS } \\
\hline 0.00 & $11.60 \mathrm{a}$ & $186.10 \mathrm{a}$ & $159.82 \mathrm{a}$ & $4.67 \mathrm{a}$ & $22.37 \mathrm{a}$ & $5.60 \mathrm{a}$ & $0.73 \mathrm{a}$ & $1.11 \mathrm{a}$ & $1.26 \mathrm{a}$ & $3.10 \mathrm{a}$ \\
\hline 7.00 & $11.00 \mathrm{a}$ & $160.00 \mathrm{a}$ & $161.35 \mathrm{a}$ & $4.50 \mathrm{a}$ & $22.72 \mathrm{a}$ & $7.78 \mathrm{a}$ & $0.79 \mathrm{a}$ & $1.23 \mathrm{a}$ & $1.23 \mathrm{a}$ & $3.26 \mathrm{a}$ \\
\hline 9.00 & $11.40 \mathrm{a}$ & $174.00 \mathrm{a}$ & $153.31 \mathrm{a}$ & $4.48 \mathrm{a}$ & $22.83 \mathrm{a}$ & $6.11 \mathrm{a}$ & $0.78 \mathrm{a}$ & $1.14 \mathrm{a}$ & $1.27 \mathrm{a}$ & $3.19 \mathrm{a}$ \\
\hline 12.00 & $10.00 \mathrm{a}$ & $161.00 \mathrm{a}$ & $134.87 \mathrm{a}$ & $4.34 \mathrm{a}$ & $22.31 \mathrm{a}$ & $5.25 \mathrm{a}$ & $0.74 \mathrm{a}$ & $1.03 \mathrm{a}$ & $1.11 \mathrm{a}$ & $2.88 \mathrm{a}$ \\
\hline 15.00 & $10.33 \mathrm{a}$ & $156.22 \mathrm{a}$ & $115.76 \mathrm{a}$ & $4.41 \mathrm{a}$ & $21.89 \mathrm{a}$ & $7.33 \mathrm{a}$ & $0.57 \mathrm{a}$ & $1.01 \mathrm{a}$ & $1.21 \mathrm{a}$ & $2.79 \mathrm{a}$ \\
\hline
\end{tabular}

Means followed by the same letter did not differ from each other in the Tukey's test at 5\% probability level. Conc: concentrations; LN: number of leaves; NL: number of leaflets; LA: leaf area; D: collar diameter; H: height; RV: root volume; SDM: stem dry mass; RDM: root dry mass; LDM: leaf dry mass; TDM: total dry mass; MSD: minimal significant difference.

Médias seguidas pela mesma letra não diferem entre si pelo teste de Tukey a 5\% de probabilidade. NF: número de folhas; NFO: número de foliolos; AF: área foliar; D: diâmetro do coleto; H: altura; VR: volume de raiz; MSC: massa seca do caule; MSR: massa seca de raiz; MSF: massa seca das folhas; MST: massa seca total; DMS: diferença mínima significativa. 
Table 3 - Mean growth values recorded for variables of seedlings belonging to species $S$. amazonicum subjected to different Hold Fire ${ }^{\circledR}$ retardant concentrations - applied on leaves.

Tabela 3 - Valores médios das variáveis de crescimento das mudas da espécie S. amazonicum, submetidas a diferentes concentrações do retardante Hold Fire ${ }^{\circledR}$-aplicadas sobre as folhas.

\begin{tabular}{|c|c|c|c|c|c|c|c|c|c|c|}
\hline Conc. & LN & NL & LA & D & $\mathbf{H}$ & RV & LDM & SDM & RDM & TDM \\
\hline & - & - & $\mathrm{cm}^{2}$ & $\mathrm{~mm}$ & $\mathrm{~cm}$ & $\mathrm{ml}$ & & & & \\
\hline & \multicolumn{10}{|c|}{15 DAT } \\
\hline 0.00 & $4.90 \mathrm{a}$ & $74.70 \mathrm{a}$ & $58.40 \mathrm{a}$ & $2.78 \mathrm{~b}$ & $13.77 \mathrm{abc}$ & $1.05 \mathrm{a}$ & $0.19 \mathrm{a}$ & $0.16 \mathrm{a}$ & $0.09 \mathrm{a}$ & $0.43 \mathrm{a}$ \\
\hline 7.00 & $5.00 \mathrm{a}$ & $69.00 \mathrm{a}$ & $60.83 \mathrm{a}$ & $2.72 \mathrm{~b}$ & $15.89 \mathrm{a}$ & $1.30 \mathrm{a}$ & $0.17 \mathrm{a}$ & $0.14 \mathrm{a}$ & $0.07 \mathrm{a}$ & $0.38 \mathrm{a}$ \\
\hline 9.00 & $3.50 \mathrm{a}$ & $58.00 \mathrm{a}$ & $63.86 \mathrm{a}$ & $2.81 \mathrm{~b}$ & $14.10 \mathrm{ab}$ & $1.15 \mathrm{a}$ & $0.17 \mathrm{a}$ & $0.13 \mathrm{a}$ & $0.08 \mathrm{a}$ & $0.38 \mathrm{a}$ \\
\hline 12.00 & $2.90 \mathrm{a}$ & $46.00 \mathrm{a}$ & $60.18 \mathrm{a}$ & $3.33 \mathrm{a}$ & $12.00 \mathrm{bc}$ & $1.40 \mathrm{a}$ & $0.16 \mathrm{a}$ & $0.12 \mathrm{a}$ & $0.08 \mathrm{a}$ & $0.36 \mathrm{a}$ \\
\hline 15.00 & $3.20 \mathrm{a}$ & $52.10 \mathrm{a}$ & $43.13 \mathrm{a}$ & $3.02 \mathrm{ab}$ & $10.43 \mathrm{c}$ & $1.10 \mathrm{a}$ & $0.12 \mathrm{a}$ & $0.10 \mathrm{a}$ & $0.06 \mathrm{a}$ & $0.28 \mathrm{a}$ \\
\hline \multirow[t]{2}{*}{ MSD } & 2.64 & 33.02 & 35.98 & 0.41 & 3.59 & 0.69 & 0.11 & 0.06 & 0.05 & 0.21 \\
\hline & \multicolumn{10}{|c|}{ 80 DAT } \\
\hline 0.00 & $9.50 \mathrm{a}$ & $127.70 \mathrm{a}$ & $57.31 \mathrm{a}$ & $4.06 \mathrm{a}$ & $14.98 \mathrm{ab}$ & $2.50 \mathrm{a}$ & $0.35 \mathrm{a}$ & $0.54 \mathrm{a}$ & $0.54 \mathrm{a}$ & $1.43 \mathrm{a}$ \\
\hline 7.00 & $10.50 \mathrm{a}$ & $134.57 \mathrm{a}$ & $53.19 \mathrm{a}$ & $3.25 \mathrm{~b}$ & $11.91 \mathrm{~b}$ & $1.12 \mathrm{~b}$ & $0.28 \mathrm{a}$ & $0.32 \mathrm{ab}$ & $0.40 \mathrm{a}$ & $1.01 \mathrm{ab}$ \\
\hline 9.00 & $11.67 \mathrm{a}$ & $169.44 \mathrm{a}$ & $58.06 \mathrm{a}$ & $3.82 \mathrm{ab}$ & $16.28 \mathrm{a}$ & $2.00 \mathrm{ab}$ & $0.35 \mathrm{a}$ & $0.52 \mathrm{a}$ & $0.51 \mathrm{a}$ & $1.38 \mathrm{a}$ \\
\hline 12.00 & $11.50 \mathrm{a}$ & $160.75 \mathrm{a}$ & $43.94 \mathrm{a}$ & $3.34 \mathrm{~b}$ & $15.07 \mathrm{a}$ & $1.25 \mathrm{~b}$ & $0.27 \mathrm{a}$ & $0.45 \mathrm{ab}$ & $0.40 \mathrm{a}$ & $1.13 \mathrm{ab}$ \\
\hline 15.00 & $10.17 \mathrm{a}$ & $140.25 \mathrm{a}$ & $39.24 \mathrm{a}$ & $3.21 \mathrm{~b}$ & $13.42 \mathrm{ab}$ & $2.92 \mathrm{a}$ & $0.24 \mathrm{a}$ & $0.24 \mathrm{~b}$ & $0.31 \mathrm{a}$ & $0.79 \mathrm{~b}$ \\
\hline MSD & 3.38 & 55.03 & 22.80 & 0.64 & 3.09 & 1.13 & 0.13 & 0.26 & 0.23 & 058 \\
\hline
\end{tabular}

Means followed by the same letter did not differ from each other in the Tukey's test at 5\% probability level. Conc: concentrations; LN: number of leaves; NL: number of leaflets; LA: leaf area; D: collar diameter; H: height; RV: root volume; SDM: stem dry mass; RDM: root dry mass; LDM: leaf dry mass; TDM: total dry mass; MSD: minimal significant difference.

Médias seguidas pela mesma letra não diferem entre si pelo teste de Tukey a 5\% de probabilidade. NF: número de folhas; NFO: número de foliolos; AF: área foliar; D: diâmetro do coleto; H: altura; VR: volume de raiz; MSC: massa seca do caule; MSR: massa seca de raiz; MSF: massa seca das folhas; MST: massa seca total;

DMS: diferença mínima significativa.

Mean RV recorded for the control concentration (0.00 $\mathrm{g} \mathrm{L}^{-1}$ of Nutrigel) did not statistically differ from any of the treatments with Nutrigel $(0.25 ; 0.50 ; 0.75$ and $\left.1.00 \mathrm{~g} \mathrm{~L}^{-1}\right)$, but this variable recorded differences between concentrations 0.50 and $0.75 \mathrm{~g} \mathrm{~L}^{-1}$. Mean
SDM recorded for seedlings that were not treated with Nutrigel $\left(0.00 \mathrm{~g} \mathrm{~L}^{-1}\right)$ was higher than the mean recorded for the ones that received 0.25 and $1.00 \mathrm{~g}$ $\mathrm{L}^{-1}$ of the polymer, but it did not differ from that of concentrated ions 0.50 and $0.75 \mathrm{~g} \mathrm{~L}^{-1}$. Mean RDM

Table 4 - Mean growth values recorded for variables of seedlings belonging to species $S$. amazonicum subjected to different waterretaining polymer Nutrigel ${ }^{\mathbb{B}}$ concentrations - applied via substrate.

Tabela 4 - Valores médios das variáveis de crescimento das mudas da espécie S. amazonicum, submetidas a diferentes concentrações do polímero hidroretentor Nutrigel ${ }^{\circledR}$ - aplicadas via substrato.

\begin{tabular}{|c|c|c|c|c|c|c|c|c|c|c|}
\hline Conc. & $\mathbf{L N}$ & $\mathbf{N L}$ & $\mathbf{L A}$ & $\overline{\mathbf{D}}$ & $\overline{\mathbf{H}}$ & $\mathbf{R V}$ & LDM & SDM & RDM & $\overline{\text { TDM }}$ \\
\hline & - & - & $\mathrm{cm}^{2}$ & $\mathrm{~mm}$ & $\mathrm{~cm}$ & $\mathrm{ml}$ & ---- & & -g--------- & -------- \\
\hline & \multicolumn{10}{|c|}{ 15 DAS } \\
\hline 0.00 & $2.40 \mathrm{a}$ & $44.40 \mathrm{a}$ & $78.08 \mathrm{a}$ & $3.33 \mathrm{a}$ & $10.41 \mathrm{a}$ & $1.90 \mathrm{ab}$ & $0.17 \mathrm{a}$ & $0.25 \mathrm{a}$ & $0.11 \mathrm{ab}$ & $0.53 \mathrm{a}$ \\
\hline 0.25 & $2.80 \mathrm{a}$ & $52.90 \mathrm{a}$ & $86.19 \mathrm{a}$ & $2.89 \mathrm{a}$ & $12.11 \mathrm{a}$ & $1.90 \mathrm{ab}$ & $0.19 \mathrm{a}$ & $0.14 \mathrm{~b}$ & $0.12 \mathrm{a}$ & $0.44 \mathrm{ab}$ \\
\hline 0.50 & $2.30 \mathrm{a}$ & $44.40 \mathrm{a}$ & $73.24 \mathrm{a}$ & $3.00 \mathrm{a}$ & $11.82 \mathrm{a}$ & $1.60 \mathrm{~b}$ & $0.16 \mathrm{a}$ & $0.18 \mathrm{ab}$ & $0.08 \mathrm{~b}$ & $0.42 \mathrm{ab}$ \\
\hline 0.75 & $2.70 \mathrm{a}$ & $52.70 \mathrm{a}$ & $78.39 \mathrm{a}$ & $2.84 \mathrm{a}$ & $12.30 \mathrm{a}$ & $2.50 \mathrm{a}$ & $0.16 \mathrm{a}$ & $0.16 \mathrm{ab}$ & $0.08 \mathrm{~b}$ & $0.41 \mathrm{ab}$ \\
\hline 1.00 & $2.70 \mathrm{a}$ & $52.00 \mathrm{a}$ & $71.45 \mathrm{a}$ & $3.10 \mathrm{a}$ & $12.08 \mathrm{a}$ & $2.10 \mathrm{ab}$ & $0.16 \mathrm{a}$ & $0.13 \mathrm{~b}$ & $0.09 \mathrm{ab}$ & $0.38 \mathrm{~b}$ \\
\hline \multirow[t]{2}{*}{ MSD } & 0.61 & 10.61 & 26.89 & 0.54 & 1.95 & 0.75 & 0.06 & 0.09 & 0.03 & 0.13 \\
\hline & \multicolumn{10}{|c|}{80 DAS } \\
\hline 0.00 & $11.6 \mathrm{a}$ & $186.1 \mathrm{a}$ & $159.82 \mathrm{a}$ & $4.67 \mathrm{a}$ & $22.37 \mathrm{a}$ & $5.60 \mathrm{a}$ & $0.73 \mathrm{a}$ & $1.11 \mathrm{a}$ & $1.26 \mathrm{a}$ & $3.10 \mathrm{a}$ \\
\hline 0.25 & $10.11 \mathrm{a}$ & $156.00 \mathrm{a}$ & $139.13 \mathrm{a}$ & $4.69 \mathrm{a}$ & $21.72 \mathrm{a}$ & $6.11 \mathrm{a}$ & $0.56 \mathrm{a}$ & $1.13 \mathrm{a}$ & $1.23 \mathrm{a}$ & $2.92 \mathrm{a}$ \\
\hline 0.50 & $9.5 \mathrm{a}$ & $138.80 \mathrm{a}$ & $157.10 \mathrm{a}$ & $4.56 \mathrm{a}$ & $23.00 \mathrm{a}$ & $6.40 \mathrm{a}$ & $0.82 \mathrm{a}$ & $1.23 \mathrm{a}$ & $1.07 \mathrm{a}$ & $3.12 \mathrm{a}$ \\
\hline 0.75 & $9.5 \mathrm{a}$ & $129.40 \mathrm{a}$ & $151.09 \mathrm{a}$ & $4.66 \mathrm{a}$ & $19.90 \mathrm{a}$ & $6.60 \mathrm{a}$ & $0.65 \mathrm{a}$ & $1.19 \mathrm{a}$ & $1.10 \mathrm{a}$ & $2.94 \mathrm{a}$ \\
\hline 1.00 & $11 \mathrm{a}$ & 169.33 & $138.31 \mathrm{a}$ & $4.21 \mathrm{a}$ & $22.67 \mathrm{a}$ & $7.33 \mathrm{a}$ & $0.73 \mathrm{a}$ & $1.12 \mathrm{a}$ & $1.03 \mathrm{a}$ & $2.88 \mathrm{a}$ \\
\hline
\end{tabular}

Means followed by the same letter did not differ from each other in the Tukey's test at 5\% probability level. Conc: concentrations; LN: number of leaves; NL: number of leaflets; LA: leaf area; D: collar diameter; H: height; RV: root volume; SDM: stem dry mass; RDM: root dry mass; LDM: leaf dry mass; TDM: total dry mass; MSD: minimal significant difference.

Médias seguidas pela mesma letra não diferem entre si pelo teste de Tukey a $5 \%$ de probabilidade. NF: número de folhas; NFO: número de foliolos; AF: área foliar: D: diâmetro do coleto; H: altura; VR: volume de raiz; MSC: massa seca do caule; MSR: massa seca de raiz; MSF: massa seca das folhas; MST: massa seca total; DMS: diferença mínima significativa.

\section{Revista Árvore 2021;45:e4514}


recorded for the control concentration did not differ from that of the four treatments with Nutrigel $(0.25$; $\left.0.50 ; 0.75 \mathrm{~g} \mathrm{~L}^{-1}\right)$; the mean recorded for this variable was higher at concentration of $0.25 \mathrm{~g} \mathrm{~L}^{-1}$ than at 0.50 and $0.75 \mathrm{~g} \mathrm{~L}^{-1}$. Variable TDM recorded higher means at the control concentration than that at the maximum hydrogel concentration $\left(1.00 \mathrm{~g} \mathrm{~L}^{-1}\right)$, but there were no differences between the control and the other concentrations $\left(0.25 ; 0.50 ; 0.75 \mathrm{~g} \mathrm{~L}^{-1}\right)$ (Table 4$)$.

There was decrease by $0.12 \mathrm{~g}(48 \%)$ in SDM accumulation and by $0.15 \mathrm{~g}(28 \%)$ in TDM in comparison to the control treatment due to the application of the highest Nutrigel ${ }^{\circledR}$ concentration (1.00 $\left.\mathrm{g} \mathrm{L}^{-1}\right)$ (Table 4).

\subsection{Nutrigel ${ }^{\circledR}$ via leaf}

The control treatment did not present differences concerning the means recorded for Nutrigel concentrations $\left(0.25 ; 0.50 ; 0.75 ; 1.00 \mathrm{~g} \mathrm{~L}^{-1}\right)$ in variables $\mathrm{LN}$ and NL, at $15 \mathrm{DAT}$. Treatment based on $0.50 \mathrm{~g} \mathrm{~L}$ did not differ from treatments with 0.25 and $0.00 \mathrm{~g} \mathrm{~L}^{-1}$, but it presented higher $\mathrm{LN}$ than concentrations 0.75 and $1.00 \mathrm{~g} \mathrm{~L}^{-1}$. Mean NL at concentration $0.50 \mathrm{~g} \mathrm{~L}^{-1}$ was only higher than that at $0.75 \mathrm{~g} \mathrm{~L}^{-1}$. Mean LA at concentration $0.50 \mathrm{~g} \mathrm{~L}^{-1}$ was higher than in treatments with 0.00 and $1.00 \mathrm{~g} \mathrm{~L}^{-1}$ of polymer. There was no significant difference between control and the other polymer applications in this variable $(0.25 ; 0.75$ and $1.00 \mathrm{~g} \mathrm{~L}^{-1}$ ) (Table 5).

$\mathrm{H}$ was higher in the treatment without the polymer (0.00) than with the maximum Nutrigel ${ }^{\circledR}$ application (1.00 $\left.\mathrm{g} \mathrm{L}^{-1}\right)$, but it was lower than the mean recorded at concentrations 0.25 and $0.50 \mathrm{~g} \mathrm{~L}^{-1}$. There were no $\mathrm{H}$ differences between means in treatments with 0.00 and $0.75 \mathrm{~g} \mathrm{~L}^{-1}$. Mean RV was higher at the minimum Hydrogel concentration $\left(0.25 \mathrm{~g} \mathrm{~L}^{-1}\right)$ than in the control treatment $(0.00)$; this treatment led to means statistically similar to those recorded at concentrations $0.50 ; 0.75$ and $1.00 \mathrm{~g} \mathrm{~L}^{-1}$. There were no differences in LDM means between the treatment without hydrogel and $1.00 \mathrm{~g} \mathrm{~L}^{-1}$; means in these treatments were lower than at Nutrigel ${ }^{\circledR}$ concentration of $0.50 \mathrm{~g} \mathrm{~L}^{-1}$ (Table 5).

The application of Nutrigel ${ }^{\circledR}$ at concentration of $0.50 \mathrm{~g} \mathrm{~L}^{-1}$ administered via substrate allowed increase by $40.04 \mathrm{~cm}^{2}(70 \%)$ in LA, by $7.7 \mathrm{~cm}(29 \%)$ in $\mathrm{H}$ and by $0.20 \mathrm{~g}(89 \%)$ in LDM accumulation at $15 \%$ DAT.

There were no significant differences between the control treatment and water-retaining polymer concentrations $\left(0.25 ; 0.50 ; 0.75 ; 1.00 \mathrm{~g} \mathrm{~L}^{-1}\right)$ applied on leaves, at 80 DAT. The difference was only observed between treatments 0.50 and $0.75 \mathrm{~g} \mathrm{~L}^{-1}$, whose SDM

Table 5 - Mean growth values recorded for variables of seedlings belonging to species $S$. amazonicum subjected to different waterretaining polymer Nutrigel ${ }^{\circledR}$ concentrations - applied on leaves.

Tabela 5 - Valores médios das variáveis de crescimento das mudas da espécie $\boldsymbol{S}$. amazonicum, submetidas a diferentes concentrações do polímero hidroretentor Nutrigel $\mathbb{R}^{\mathbb{E}}$ - aplicadas sobre as folhas.

\begin{tabular}{|c|c|c|c|c|c|c|c|c|c|c|}
\hline Conc. & $\overline{\mathbf{L N}}$ & $\mathbf{N L}$ & $\mathbf{L A}$ & $\overline{\mathbf{D}}$ & $\overline{\mathbf{H}}$ & $\mathbf{R V}$ & LDM & SDM & RDM & $\overline{\text { TDM }}$ \\
\hline & - & - & $\mathrm{cm}^{2}$ & $\mathrm{~mm}$ & $\mathrm{~cm}$ & $\mathrm{ml}$ & ----- & . & g------- & ------- \\
\hline & \multicolumn{10}{|c|}{15 DAT } \\
\hline 0.00 & $4.90 \mathrm{ab}$ & $74.70 \mathrm{ab}$ & $58.40 \mathrm{~b}$ & $2.78 \mathrm{a}$ & $13.77 \mathrm{~b}$ & $1.05 \mathrm{~b}$ & $0.18 \mathrm{~b}$ & $0.16 \mathrm{a}$ & $0.09 \mathrm{a}$ & $\overline{0.43 \mathrm{a}}$ \\
\hline 0.25 & $4.40 \mathrm{ab}$ & $71.30 \mathrm{ab}$ & $88.73 \mathrm{ab}$ & $3.02 \mathrm{a}$ & $19.03 \mathrm{a}$ & $1.70 \mathrm{a}$ & $0.27 \mathrm{ab}$ & $0.23 \mathrm{a}$ & $0.15 \mathrm{a}$ & $0.65 \mathrm{a}$ \\
\hline 0.50 & $5.60 \mathrm{a}$ & $88.80 \mathrm{a}$ & $99.13 \mathrm{a}$ & $2.99 \mathrm{a}$ & $17.77 \mathrm{a}$ & $1.60 \mathrm{ab}$ & $0.34 \mathrm{a}$ & $0.22 \mathrm{a}$ & $0.15 \mathrm{a}$ & $0.71 \mathrm{a}$ \\
\hline 0.75 & $2.80 \mathrm{~b}$ & $52.80 \mathrm{~b}$ & $79.66 \mathrm{ab}$ & $2.91 \mathrm{a}$ & $11.42 \mathrm{bc}$ & $1.50 \mathrm{ab}$ & $0.21 \mathrm{ab}$ & $0.13 \mathrm{a}$ & $0.11 \mathrm{a}$ & $0.46 \mathrm{a}$ \\
\hline 1.00 & $2.90 \mathrm{~b}$ & $46.00 \mathrm{ab}$ & $59.09 \mathrm{~b}$ & $2.91 \mathrm{a}$ & $10.07 \mathrm{c}$ & $1.20 \mathrm{ab}$ & $0.14 \mathrm{~b}$ & $0.17 \mathrm{a}$ & $0.15 \mathrm{a}$ & $0.46 \mathrm{a}$ \\
\hline \multirow[t]{2}{*}{ MSD } & 2.46 & 36.13 & 38.28 & 0.54 & 3.03 & 0.64 & 0.15 & 0.11 & 0.17 & 0.31 \\
\hline & \multicolumn{10}{|c|}{80 DAT } \\
\hline 0.00 & $9.50 \mathrm{a}$ & $127.70 \mathrm{a}$ & $57.31 \mathrm{a}$ & $4.06 \mathrm{a}$ & $14.98 \mathrm{a}$ & $2.50 \mathrm{a}$ & $0.35 \mathrm{a}$ & $0.54 \mathrm{ab}$ & $0.54 \mathrm{a}$ & $1.43 \mathrm{ab}$ \\
\hline 0.25 & $9.13 \mathrm{a}$ & $112.88 \mathrm{a}$ & $62.98 \mathrm{a}$ & $4.13 \mathrm{a}$ & $18.00 \mathrm{a}$ & $4.41 \mathrm{a}$ & $0.38 \mathrm{a}$ & $0.66 \mathrm{ab}$ & $0.81 \mathrm{a}$ & $1.85 \mathrm{ab}$ \\
\hline 0.50 & $8.56 \mathrm{a}$ & $108.33 \mathrm{a}$ & $42.38 \mathrm{a}$ & $3.37 \mathrm{a}$ & $16.28 \mathrm{a}$ & $4.22 \mathrm{a}$ & $0.26 \mathrm{a}$ & $0.47 \mathrm{~b}$ & $0.53 \mathrm{a}$ & $1.26 \mathrm{~b}$ \\
\hline 0.75 & $6.50 \mathrm{a}$ & $89.00 \mathrm{a}$ & $65.07 \mathrm{a}$ & $3.50 \mathrm{a}$ & $18.00 \mathrm{a}$ & $4.67 \mathrm{a}$ & $0.39 \mathrm{a}$ & $0.84 \mathrm{a}$ & $0.76 \mathrm{a}$ & $1.99 \mathrm{a}$ \\
\hline 1.00 & $9.00 \mathrm{a}$ & $116.75 \mathrm{a}$ & $57.32 \mathrm{a}$ & $3.44 \mathrm{a}$ & $18.30 \mathrm{a}$ & $4.10 \mathrm{a}$ & $0.36 \mathrm{a}$ & $0.61 \mathrm{ab}$ & $0.59 \mathrm{a}$ & $1.56 \mathrm{ab}$ \\
\hline MSD & 4.00 & 53.73 & 25.37 & 0.81 & 3.97 & 2.39 & 0.16 & 0.32 & 0.32 & 071 \\
\hline
\end{tabular}

Means followed by the same letter did not differ from each other in the Tukey's test at 5\% probability level. Conc: concentrations; LN: number of leaves; NL: number of leaflets; LA: leaf area; D: collar diameter; H: height; RV: root volume; SDM: stem dry mass; RDM: root dry mass; LDM: leaf dry mass; TDM: total dry mass; MSD: minimal significant difference.

Médias seguidas pela mesma letra não diferem entre si pelo teste de Tukey a 5\% de probabilidade. NF: número de folhas; NFO: número de folíolos; AF: área foliar, D: diâmetro do coleto; H: altura; VR: volume de raiz; MSC: massa seca do caule; MSR: massa seca de raiz; MSF: massa seca das folhas; MST: massa seca total DMS: diferença mínima significativa. 
and TDM means were higher at the mean polymer concentration $\left(0.50 \mathrm{~L}^{-1}\right)$ (Table 5).

\section{DISCUSSION}

\subsection{Phos-Check WD881 ${ }^{\circledR}$}

The tested Phos-Chek WD881 ${ }^{\circledR}$ retardant concentrations did not have any negative effect on the growth rates recorded for species $S$. amazonicum. Variation in the forms of contact between retardant and seedlings led to different responses. Contact via substrate did not cause any difference in seedlings' growth, whereas application via leaf at the highest tested Phos-Chek WD881 ${ }^{\circledR}$ concentration $(10.00 \mathrm{~mL}$ $\left.\mathrm{L}^{-1}\right)$ led to positive effects on LA (15 DAT), H (15 and 80 DAT) and TDM (80DAT) growth in comparison to the control. Mean NL, LA, D, H, RV, LDM, SDM and RDM (80 DAT) values in at least one of the PhosChek WD881 ${ }^{\circledR}$ concentrations via leaf were higher than, or equal to, that of treatments without retardant; this finding has evidenced that this product does not disturb the growth dynamics of $S$. amazonicum seedlings.

Song et al. (2014) also assessed Phos-Chek WD $881^{\circledR}$ concentrations and found that this retardant does not affect the survival of forest species such as Quarcus acutíssima, Quarcus palustres and of herbaceous species Brassica campestris. There were no changes in these species even at doubled application rates. Based on the physiological responses measured by these authors (chlorophyll content and enzyme superoxide dismutase), Phos-Chek does not pose any long-term phytotoxic risk.

Lack of Phos-Chek WD881 ${ }^{\circledR}$ (via substrate) influence on the growth parameters of $S$. amazonicum seedlings points towards the fact that different inorganic chemical molecules composing the retardant were not metabolized by plant cells. In case these molecules have entered the organism, such substances were likely partitioned into storage organelle cells or, yet, became biochemically inactive. On the other hand, most Phos-Chek WD881 ${ }^{\circledR}$ compounds are surfactant substances likely absorbed by substrate particles. The absorption of surfactant molecules in the soil depends on the nature of such a surfactant and on soil physicalchemical properties (Sánchez-Martín et al., 2008).

Results in the current study corroborate that by Hartskeerl et al. (2004), who assessed the growth responses of Australian plant species after retardant foam application and did not find any response from such a substance in any of the 7 assessed species (Hardenbergia violacea (Schneev) Stern, Indigofera australis Willd., Acaciamelanoxylon R.Br., Eucalyptus polyanthemos Schauer., Banksia integrifolia L.f., Grevillea sp., Poa labillardierei Steud.). It is important highlighting that the concentrations adopted in their research were also diluted based on the application limits recommended by the manufacturer. Not even species sensitive to environmental changes (Family: Proteaceae) showed any negative effects in comparison to the application of the short duration retardant - such a finding has evidenced that this product does not pose any phytotoxic risk when it is used according to the limits set by the manufacturer. According to Hartskeerl et al. (2004), foam impacts partially depend on habitat type and environmental conditions; moreover, they are relatively small, since no harming effect on vegetation was observed at the end of 14 weeks.

The growth observed in the shoots of $S$. amazonicum seedlings due to the application of the highest Phos-Chek WD881 ${ }^{\circledR}\left(10.00 \mathrm{~mL} \mathrm{~L}^{-1}\right)$ concentration on leaves (15 and 80 DAT) is likely related to the development of a liquid surface formed by the retardant in the water-air interface, in leaves. Surfactants found in this retardant reduced water surface stress and led to hydrogen bridge disruption; this process changed the shape of water drops and allowed the widespread and penetration of the liquid in different surfaces easier, for example, in leaf blade (Appah et al., 2019; Gao et al., 2019). Thus, liquid water bubbles emerge, maximize the wet surface area and impair leaf transpiration.

Results recorded for Phos-Chek WD881 ${ }^{\circledR}$ application in the present study corroborated the study by Song et al., (2014), according to whom, plants recover from stress caused by short duration retardants and these retardants mean no danger of phytotoxic effect on the long-run.

\subsection{Hold Fire ${ }^{\circledR}$}

The influence of retardant Hold Fire ${ }^{\circledR}$ on growth rates recorded for $S$. amazonicum seedlings has changed due to concentration $(7.00 ; 9.00 ; 12.00$ or $15.00 \mathrm{ml} \mathrm{L}^{-1}$ ) and application form (via substrate or leaf). Retardant interaction with seedlings via

Revista Árvore 2021;45:e4514 
substrate did not cause any negative change in their growth if one takes into account values observed for the shoot of $S$. amazonicum seedlings (15 DAS and 15 DAT). Hold Fire ${ }^{\circledR}$ has natural hygroscopic polymers, i.e., polymers capable of absorbing water. This feature explains the similitude between means recorded for seedlings that have received water and the ones that were treated with retardant, except for concentration of $12.00 \mathrm{ml} \mathrm{L}^{-1}$, which led to higher $\mathrm{H}$ value than that of seedlings treated with water (control).

Mean H, RV (15 DAS) and D, H (15 DAT) in the treatment without Hold Fire ${ }^{\circledR}\left(0.00 \mathrm{ml} \mathrm{L}^{-1}\right)$ were statistically lower, or equal to, at least one of the treatments added with retardant $(7.00 ; 9.00 ; 12.00$ or $\left.15.00 \mathrm{ml} \mathrm{L}^{-1}\right)$; in other words, this product presents effects similar to that of water in the first 15 plant development days. Plantlets get totally vulnerable to biotic and abiotic factors at the initial establishment phase, mainly to water availability (Hadi et al., 2018; Luo et al., 2015), given the small size of their roots and their fast cell development. Hold Fire ${ }^{\circledR}$ has surfactants and, according to Moore et al. (2010), some surfactants enhance soil wettability and increase water availability to plants; consequently, they favor plant growth.

The negative effect observed at 80 DAT on the diameter growth process, stem dry mass and total dry mass accumulation in $S$. amazonicum seedlings due to the application of the highest Hold Fire ${ }^{\circledR}$ concentration on leaves is likely related to the action of surfactant's fat-soluble inorganic molecules on leaves. Some surfactants change the physical-chemical properties of leaf surface, mainly of leaf epidermis, by corroding the protective wax and the cuticles. Damato et al. (2017) observed epicuticular wax dissolution in soybean leaves due to adjuvant actions. Leaf cuticle has lipophilic and hydrophilic compounds whose main function lies on avoiding water loss (Fernandez et al., 2017). The photosynthetic activity is reduced by the surfactant's action in the waxy layer of leaves (Räsch et al., 2018), a fact that influences plant growth.

It was possible observing that such an effect derives from successive Hold Fire ${ }^{\circledR}$ applications at 80 DAT. There was greater diameter growth in seedlings treated with $12.00 \mathrm{~mL} \mathrm{~L}^{-1}$ of Hold Fire ${ }^{\circledR}$ at 15 DAT to the detriment of the ones that only received water (control treatment). Hygroscopic polymer's ability to keep water availability to leaves seems to fail against the effects of surfactant application on leaves because of the successive applications of it. Therefore, besides determining safe doses of this substance, it is necessary being careful with the number of Hold Fire ${ }^{\circledR}$ applications within the same area. Overall, places facing recurrent fire events become environmentally sensitive and may house rare or endangered species (Hartskeerl et al., 2004).

\subsection{Nutrige ${ }^{\circledR}$}

Based on results in Table 4, the water-retaining polymer Nutrigel ${ }^{\circledR}$ did not cause any permanent disturbance in the growth of $S$. amazonicum representatives. Assumingly, the negative effect of it on seedlings' SDM and TDM at 15 DAS was a genetic feature of the species itself. Seedlings were treated with $1.00 \mathrm{~g} \mathrm{~L}^{-1}$ of Nutrigel ${ }^{\circledR}$ via substrate since sowing. Keffer (2019) assessed the effects of this polymer on the germination and vigor of $S$. amazonicum seedlings and found reduced number of normal plantlets due to Nutrigel ${ }^{\circledR}$ application. On the other hand, they found larger numbers of them in T. roseoalba, H. impetiginosus and E. schomburgkii seedlings subjected to the same concentrations of this water-retaining polymer.

According to Keffer (2019), the water-potential difference between Nutrigel and seedlings has likely guided the water flow into the seeds; this process led to the formation of abnormal plantlets. Lower SDM and TDM in seedlings may be the reflex of such a prior disorder in the seed. S. amazonicum is a pioneer species highly capable of adapting to stress as seen at 80 DAS; none of the growth variables was influenced by Nutrigel application via substrate.

The main goal of adopting the treatment with hydrogel lied on reaching higher efficiency in using water to produce forest seedlings in plant nursery (Navroski, et al., 2015). These benefits boosted the use of water-retaining polymer as flame retardants. The literature addresses several examples of tree species such as Eucalyptus dunnii (Navroski et al., 2015) Handroanthus ochraceus (Mews et al., 2015), coffee (Souza et al., 2016), Corymbia citriodora (Azevedo et al., 2016), Cordia trichotoma (Kelling, et al., 2017), Mimosa scabrella (Kozen et al., 2017) and Enterolobium contortisilliquum (Pontes Filho et al., 
2018) that have potentiated their own growth after the application of water-retaining polymers via substrate.

Based on the results, $S$. amazonicum seedlings can benefit from small aggregates of water-retaining polymers on leaves. The treatment based on $0.50 \mathrm{~g}$ $\mathrm{L}^{-1}$ of Nutrigel ${ }^{\circledR}$ via leaf led to higher LA, H and LDM (15 DAT) means than water application (0.00). The other growth variables (LN, NL, RV at 15 DAT; SDM and TDM at 80 DAT) recorded for seedlings that have received some Nutrigel concentration were the same as those of the control. However, there is no record in the literature about hydrogel application on plants' leaves.

Leaves are the main organs involved in dry mass production due to photosynthesis (Tondjo et al., 2015). One can infer that Nutrigel does not have adverse effect on vegetation when it is applied according to the recommended concentrations because it is consolidated in the agroforest market, whose goal is to boost plant growth through water availability. Hydrogel contact on leaf surface impaired the soil/plant interaction, led to greater leaf hydration and contributed to lower leaf temperature. Leaf temperature influenced gas exchange and had direct effect on photosynthesis and respiration. Many authors have seen high leaf temperatures as the main factor harming carbon fixation, since they affect enzyme activity (Heskel et al., 2016; Slot and Winter, 2017; Blonder and Michaletz, 2018).

\section{CONCLUSIONS}

Phos-Chek WD88 $1{ }^{\circledR}$ concentrations of $3.00,6.00$, 8.00 and $10.00 \mathrm{~mL} \mathrm{~L}^{-1}$ applied via substrate on leaves did not impair the initial growth of $S$. amazonicum seedlings. The highest concentration of it $(10.00 \mathrm{~mL}$ $\mathrm{L}^{-1}$ ) applied on leaves has favored shoot growth in seedlings.

The initial growth of $S$. amazonicum representatives was not negatively affected by Hold Fire ${ }^{\circledR}$ concentrations of 7.00, 9.00, 12.00 and $15.00 \mathrm{~mL}^{-1}$ applied via substrate. The Hold Fire ${ }^{\circledR}$ concentration of $12.00 \mathrm{~mL} \mathrm{~L}^{-1}$ applied via substrate has favored height increment in $S$. amazonicum seedlings.

Longer depositions of the highest Hold Fire ${ }^{\circledR}$ (15.00 $\mathrm{mL} \mathrm{L}^{-1}$ ) concentrations applied on leaves harmed S. amazonicum growth in diameter, stem dry mass accumulation and total $S$. amazonicum with time.

The application of water-retaining polymer Nutrige $\mathrm{l}^{\circledR}\left(0.25 ; 0.50 ; 0.75 ; 1.00 \mathrm{~g} \mathrm{~L}^{-1}\right)$ as retardant via substrate did not cause any long-term harming effect on the initial growth of $S$. amazonicum representatives. The Nutrigel ${ }^{\circledR}$ concentration of $0.50 \mathrm{~g} \mathrm{~L}^{-1}$ applied on leaves had positive effect on shoot growth in $S$. amazonicum seedlings.

Given the Brazilian heterogeneity and floristic complexity, it is important highlighting the relevance of further studies to be carried out in different environments, as well as the assessment of other plant species, so that fire retardant effects on plants can be clarified and safe environmental concentrations normalized.

\section{AUTHOR CONTRIBUTIONS}

Elen Silma Oliveira Cruz Ximenes: Data curation, Formal Analysis, Investigation, Validation, Project administration, Visualization, Writing - original draft, Writing - review \& editing.

Andréa Carvalho da Silva: Conceptualization, Project administration, Funding acquisition, Supervision, Writing - review \& editing.

Adilson Pacheco de Souza: Conceptualization, Project administration, Funding acquisition, Supervision, Writing - review \& editing.

Josiane Fernandes Keffer: Data curation, Conceptualization, Methodology, Investigation

Alison Martins dos Anjos: Data curation, Investigation.

Felipe Gomes Costa: Data curation, Investigation.

\section{REFERENCES}

Appah S, Zhou H, Wang P, Ou M, Jia W. Charged monosized droplet behaviour and wetting ability on hydrophobic leaf surfaces depending on surfactantpesticide concentrate formulation. Journal of Electrostatics. 2019;100:103356. doi:10.1016/j. elstat.2019.103356.

Aragão LEOC, Anderson LO, Fonseca MG, Rosan TM, Vedovato L, Wagner F, et al. 21st Century drought-related fires counteract the decline of

Revista Árvore 2021;45:e4514 
Amazon deforestation carbon emissions. Nature Communications. 2018;9(1). doi: 10.1038/s41467017-02771-y

Azevedo GTOS, Azevedo GB, Souza AM, Mews C L, Sousa JRL. Effect of hydrogel doses in the quality of Corymbia citriodora Hill \& Johnson seedlings. Nativa. 2016;4(4): 244-248. doi: 10.14583/23187670.v04n04a10

Blonder B, Michaletz ST. A model for leaf temperature decoupling from air temperature. Agricultural and Forest Meteorology. 2018;262:354360. doi:10.1016/j.agrformet.2018.07.012.

Canzian WP, Fiedler NC, Brinate IB, Juvanhol RS, Bighi KN. Diferent concentrations of fire retardant in eucalyptus plantations. Nativa. 2016;4(4):195-198. doi:10.14583/2318-7670.v04n04a02

Cordeiro IMCC, Barros PLC, Lameira OA, Filho ABG. Assessment of paricá (Schizolobium parahyba var.amazonicum (Huber ex Ducke) Barneby) plantations at different ages and cultivation systems in Aurora do Pará (Pará stateBrazil). Ciência Florestal. 2015;25(3):679-687. doi:10.5902/1980509819618

Couto-Vazquéz A, Gonzaléz-Prieto SJ. Long-term effects of fire and three fire-fighting chemicals on a soil-plant system. Braña. 2013;11(1):01-25.

Damato TC, Carrasco LDM, Carmona-Ribeiro AM, Luiz RV, Godoy R, Petri DFS. The interactions between surfactants and the epicuticular wax on soybean or weed leaves: maximal crop protection with minimal wax solubilization. Crop Protection. 2017;91:57-65. doi:10.1016/j.cropro.2016.09.019

Fernandez V, Bahamonde HA, Peguero-Pina JJ, Gil-Pelegrin E, Sancho-Knapik D, Gil L, et al. Physico-chemical properties of plant cuticles and their functional and ecological significance. Journal of Experimental Botany. 2017;68(19):5293-5306. doi: $10.1093 / \mathrm{jxb} / \mathrm{erx} 302$

Fiedler NC, Canzian WP, Mafia RG, Ribeiro GA, Junior JK. Intensity of burning flame in different fire retardants. Revista Árvore. 2015;39(4):691-696. doi:10.1590/0100-67622015000400011

Gao Y, Lu J, Zhang P, Shi G, Li Y, Zhao J, et al. Wetting and adhesion behavior on apple tree leaf surface by adding different surfactants. Colloids and Surfaces B: biointerfaces. 2019;110602. doi:10.1016/j.colsurfb.2019.110602

Giménez A, Pastor E, Zárate L, Planas E, Arnaldos J. Long-term forest fire retardants: a review of quality, effectiveness, application and environmmental considerations. International Journal of Wildland Fire. 2004;13:1-15. doi:10.1071/WF03001

Hadi SMS, Ahmed MZ, Hameed A, Khan MA, Gul, B. Seed germination and seedling growth responses of toothbrush tree (Salvadora persica Linn.) to different interacting abiotic stresses. Flora. 2018; 243:45-52. doi:10.1016/j.flora.2018.04.002

Hartskeerl K, Simmons D, Adams R. Does firefighting foam affect the growth of some Australian native plants. International Journal of Wildland Fire. 2004;(13):335-341. doi:10.1071/ WF03065

Heskel MA, O'Sullivan O, Reich PB, Tjoelker MG, Weerasinghe LK, Penillard A, et al. Convergence in the temperature response of leaf respiration across biomes and plant functional types. Proceedings of the National Academy of Sciences. 2016;113(14):38323837. doi:10.1073/pnas.1520282113

Kalabokidis KD. Effects of wildfire suppression chemicals on people and the environment- a review. Global Nest Journal. 2000;2(1):129-137. doi:10.30955/gnj.000144

Keffer, J. F. Effect of fire retardants on germination of tropical forest species [dissertation]. Sinop: Universidade Federal de Mato Grosso; 2019.

Kelling MB, Araujo MM, León EB, Aimi SC, Turcheto F. Irrigation regime and water retaining polymer doses in morphological and physiological characteristics of Cordia trichotoma seedlings. Bosque. 2017;38(1):123-131. doi:10.4067/S071792002017000100013

Kozen ER, Navroski MC, Friederichs G, Ferrari LH, Pereira MO, Felippe D. The use of hydrogel combined with appropriate substrate and fertilizer improve quality and growth performance of Mimosa scabrella Benth. Seedlings. Cerne. 2017;23(4):473482. doi:10.1590/01047760201723042440

Lima DC, Souza AP, Keffer JF, Bouvié L, Martim CC, Ferneda BF. Effectiveness of water-retaining Polymer as fire retardant in indirect use. Revista 
Árvore. 2020a;44;e4493. doi: 10.1590/1806908820200000029

Lima DC, Souza AP, Cabeceira FG, Keffer JF, Pizzato M, Borella DR. Volume de calda e concentração de retardantes de fogo em queimas controladas em área de eucalipto na transição Cerrado-Amazônia. Ciência Florestal. 2020b;30(1):205-220. doi: 10.5902/1980509838583

Luo HH, Zhang YL, Zhang WF. Effects of water stress and rewatering on photosynthesis, root activity, and yield of cotton with drip irrigation under mulch. Photosynthetica. 2015;54(1):65-73. doi:10.1007/ s11099-015-0165-7

Machado Filho C, Martins MC, Ribeiro GA, Lima GS, Cardoso MT, Torres CMME, Pinto FB. Efficiency of a long-term retardant used in forest fire. Ciência Florestal. 2012;22(2):365-371. doi: $10.5902 / 198050985743$

Marshall A, Waller L, Lekberg AY. Cascading effects of fire retardant on plant-microbe interactions,community composition, and invasion. Ecological Applications. 2016;26(4):996-1002. doi:10.1890/16-0001.1

Mews CL, Sousa JRL, Azevedo GTOS, Souza AM. Effect of hydrogel and urea on the seedling production of Handroanthus ochraceus (Cham.) Mattos. Floresta e Ambiente. 2015;22(1):107-116. doi:10.1590/2179-8087.080814

Moore D, Kostka SJ, Boerth TJ, Franklin M, Ritsema CJ, Dekker LW, et al. The effect of soil surfactants on soil hydrological behavior, the plant growth environment, irrigation efficiency and water conservation. Journal of hydrology and hydromechanics. 2010;58(3):142-148. doi:10.2478/ v10098-010-0013-1

Navroski MC, Araújo MM, Reininger LRS, Muniz MFB, Pereira MO. Influence of hydrogel on growth and nutritional content of Eucalyptus dunnii seedlings. Floresta. 2015;45(2):315-328.

Pontes Filho RA, Gondim FA, Costa MCG. Seedling growth of tree species under doses of hydrogel and two levels of luminosity. Revista Árvore. 2018;42(1):e420112. doi:10.1590/180690882018000100012

Plucinski MP, Sullivan AL, Hurley RJ. A methodology for comparing the relative effectiveness of suppressant enhancers designed for the direct attack of wildfires. Fire Safety Journal.2017;87:71-79. doi:10.1016/j. firesaf.2016.12.005

Räsch A, Hunsche M, Mail M, Burkhardt J, Noga G, Pariyar S. Agricultural adjuvants may impair leaf transpiration and photosynthetic activity. Plant Physiology and Biochemistry. 2018;132:229-237. doi:10.1016/j.plaphy.2018.08.042>.

Sánchez-Martín MJ, Dorado MC, Hoyo C, Rodríguesz-Cruz MS. Influence of clay mineral structure and surfactant nature on the adsorption capacity of surfactants by clays. Journal of Hazardous Materials. 2008;150:115-123. doi:10.1016/j.jhazmat.2007.04.093

Silva SS, Fearnside PM, Graça PMLA, Brown IF, Alencar A, Melo AWF. Dynamics of forest fires in the southwestern Amazon. Forest Ecology and Management. 2018;424:312-322. doi:10.1016/j. foreco.2018.04.041

Slot M, Winter K. In situ temperature response of photosynthesis of 42 tree and liana species in the canopy of two Panamanian lowland tropical forests with contrasting rainfall regimes. New Phytologist. 2017;214(3):1103-1117. doi:10.1111/nph.14469

Song U, Mun S, Waldman B, Lee EJ. Effects of three fire-suppressant foams on the germination and physiological responses of plants. Environmental Management. 2014;54:865-874. doi: 10.1007/ s00267-014-0303-1

Souza AP, Mota LL, Zamadei T, Martim CC, Almeida FT, Paulino J. Climate classification and climatic water balance in Mato Grosso state, Brazil. Nativa. 2013;1:34-43. doi:10.14583/2318-7670. v01n01a07

Souza HN, Araújo TG, Ribeiro GA. Efficyency assessemnt of the hidroretentor gel as fire retardant. Revista Árvore. 2012;36(3):471-477. doi:10.1590/ S0100-67622012000300009

Souza AJ, Guimarães RJ, Dominguetti AW, Scalco MS, Rezende TT. Water-retaining polymer and seedling type when planting irrigated coffee. Revista Ciência Agronômica. 2016;47(2):334-343. doi:10.5935/1806-6690.20160039

Tondjo K, Brancheriau L, Sabatier SA, Kokutse

\section{Revista Árvore 2021;45:e4514}


AD, Akossou A, Kokou K, et al. Non-destructive measurement of leaf area and dry biomass in Tectona grandis. Trees. 2015;29(5):1625-1631. doi:10.1007/ s00468-015-1227-y 